\title{
Metas de socialização da emoção: um estudo de mães residentes no meio rural
}

\author{
Bianca Reis Fonseca \\ Lília Iêda Chaves Cavalcante \\ Universidade Federal do Pará, PA, Brasil \\ Deise Maria Leal Fernandes Mendes \\ Universidade do Estado do Rio de Janeiro, RJ, Brasil
}

\begin{abstract}
Resumo
Este estudo investigou as metas de socialização da emoção de 40 mães de crianças, com idade entre 1 e 35 meses, residentes num município do interior do estado do Pará. Foram utilizados os seguintes instrumentos para a coleta de dados: Formulário de Identificação dos Participantes (FIP), Formulário de Dados Sociodemográficos (FDSD) e Questionário de Metas de Socialização da Emoção (QMSE). Empregou-se a análise temática categorial para o tratamento dos dados do questionário. As frequências de respostas dos participantes para cada categoria e os dados sociodemográficos foram analisados de maneira descritiva. Constatou-se que a maioria das mães mencionou metas relacionadas à automaximização $(47,7 \%)$ e à emotividade $(19,3 \%)$. Além disso, a maior parte destas ressaltou a preocupação de exercer um papel ativo no desenvolvimento emocional dos filhos $(44,7 \%)$, contribuindo diretamente para que a criança adquira características emocionais desejadas a partir do aconselhamento e exemplos diários (69,7\%). Tais resultados sugerem que as mães, no meio rural, permanecem na companhia da criança durante um longo período de sua rotina diária, por isso, tendem a transmitir com mais intensidade certas emoções (felicidade, amabilidade e afetividade ao próximo), além de auxiliar na compreensão das experiências emocionais vivenciadas na infância. Nessa lógica, constata-se que as etnoteorias parentais prototípicas do contexto rural influenciam as mães na definição das metas de socialização da emoção, e, por esta razão, sugere-se novos estudos que possam captar suas permanências e mudanças.
\end{abstract}

Palavras-chave: Metas de socialização; Desenvolvimento emocional infantil; Mães.

\section{Emotional socialization goals: a research with mothers residing in rural areas}

Abstract

This study investigated the emotional socialization goals in 40 mothers of children with 1 to 35 months aged, resident in a county of Pará State. The following instruments were used to data collect: Participants Identification Form (FIP), Sociodemographic Data Form (FDSD) and Emotional Socialization Goals Questionnaire (QMSE). A categorical thematic analysis was implemented, as support to the questionnaire data. The frequencies of responses for each category and the sociodemographic data, were analyzed through a descriptive method. It was verified that the majority of mothers mentioned goals related to automaximization (47.7\%) and emotivity (19.3\%). Moreover, the majority emphasized their concern to play an active role in children's emotional development $(44.7 \%)$, directly contributing to the child's acquisition of desired emotional characteristics from daily advices and examples $(69.7 \%)$. These results suggest that mothers of rural environment remain with their children for a long period of their daily routine. Therefore, these mothers tend to transmit certain emotions (e.g.happiness, kindness and affection to others) more intensely, besides helping in comprehension of emotional and socialization goals. For this reason, new investigations might be necessary, in order to capture permanences and changes of such behaviors.

Keywords: Socialization goals; Child's emotional development; Mothers.

\section{Objetivos de socialización emocional: una investigación con madres residentes en áreas rurales}

\section{Resumen}

Este estudio investigó los objetivos de socialización emocional en 40 madres, con niños de 1 a 35 meses de edad, residentes en un condado del estado de Pará. Fueron utilizados los siguientes instrumentos para la obtención de datos: Formulario de identificación de participantes (FIP), Formulario de datos sociodemográficos (FDSD) y Cuestionario de metas de socialización emocional (QMSE). Un análisis temático categórico fue implementada, como apoyo a los datos del cuestionario. Las frecuencias de las respuestas para cada categoría y los datos sociodemográficos, se analizaron por médio de un procedimiento descriptivo. Así, se comprobó que la mayoría de las madres mencionaron objetivos relacionados con la automaximización (47,7\%) y emotividad $(19,3 \%)$. Por otra parte, la mayoría enfatizó su preocupación por desempeñar un papel activo en el desarrollo emocional de los niños $(44,7 \%)$, contribuyendo directamente a la adquisición de características emocionales deseadas por parte del niño a partir de consejos y ejemplos diarios $(69,7 \%)$. Estos resultados sugieren que las madres del medio rural permanecen con sus hijos durante un largo período de su rutina diaria. Por lo tanto, estas madres tienden a transmitir más intensamente ciertas emociones (por ejemplo felicidad, bondad y afecto a los demás), además de ayudar a la comprensión de los objetivos emocionales y de socialización. Por esta razón, podrían ser necesarias nuevas investigaciones para captar las permanencias y cambios de tales comportamientos.

Palabras clave: Objetivos de socialización; Desarrollo emocional del niño; Madres. 
O desenvolvimento emocional na primeira infância contribui para uma série de habilidades que se associam entre si, e tornam mais complexas possíveis aquisições cognitivas e relações interpessoais. Nesse sentido, o desenvolvimento da capacidade de regular as emoções em situações desafiadoras, por exemplo, favorece a aprendizagem da criança e a comunicação dos próprios sentimentos ao interagir com os outros, além de facilitar a compreensão do que eles estão sentindo. A capacidade de regular as emoções permite que a criança modifique seu comportamento de modo a manter harmoniosamente suas relações interpessoais, e torna a interação social mais eficaz (Halberstadt \& Lozada, 2011).

Embora as emoções consideradas básicas possam ser vistas como fenômenos universais (Ekman \& Davidson, 1994), a cultura desempenha um papel importante ao distinguir o significado emocional frente a eventos sociais (Cole \& Tamang, 1998), determinando como se expressar e agir diante de situações específicas (Keller \& Otto, 2009; Mendes \& Cavalcante, 2014). Desse modo, teóricos e pesquisadores defendem que, para a melhor compreensão dos vários aspectos implicados no desenvolvimento emocional, torna-se importante considerar as crenças e práticas parentais relacionadas à expressão emocional e à comunicação afetiva com base no contexto em que elas ocorrem (Halberstadt \& Lozada, 2011; Ho, 2014; Mendes \& Cavalcante, 2014; Mendes \& Pessôa, 2013). Parte-se da convicção de que a parentalidade tem sido considerada como uma característica significativa da cultura, e um importante mecanismo para a transmissão de valores culturais e práticas entre as gerações (Harkness \& Super, 1996; Ho, 2014; Keller, 2007).

$\mathrm{Na}$ esteira dessas preocupações, Harkness e Super $(1992,1994)$ elaboraram o modelo de Nicho de Desenvolvimento como uma opção teórica para a compreensão contextualizada do desenvolvimento, inclusive em sua dimensão emocional, pois considera a criança e o ambiente como um sistema interativo. Desse modo, admite-se que haja uma interação contínua entre três componentes ou subsistemas do modelo: o ambiente físico e social da criança, os costumes culturalmente construídos sobre os cuidados e modos de criá-la e a psicologia dos que cuidam. No subsistema do ambiente físico e social, encontram-se as fontes mais diretas de informação sobre como o ambiente social da criança é estruturado. Nele, observa-se com quem e como ela vive, e ainda em que condições materiais. Os costumes e os modos próprios de cuidar das crianças constituem outro subsistema importante, que, na perspectiva do Nicho Desenvolvimental, informa sobre as práticas comuns de cada grupo na criação e no cuidado destas.
A psicologia dos cuidadores corresponde ao terceiro subsistema, que compreende crenças e expectativas daqueles que são responsáveis pelos cuidados às crianças (Harkness \& Super, 1992; 1994).

A psicologia dos cuidadores está presente nas atividades cotidianas, nos julgamentos, escolhas e decisões que estes são levados a tomar em relação a seus filhos (Harkness \& Super 1996). Dessa maneira, crenças e valores dos cuidadores podem ser definidos como etnoteorias parentais, que, por sua vez, informam os domínios representacionais da parentalidade (Keller \& Kärtner, 2013) nos quais estão incluídas as metas de socialização que os pais traçam para o futuro dos filhos e as práticas diárias que eles julgam importantes para atingi-las (Martins, 2014).

Em uma investigação que também comparava culturas diversas, Camras, Oster, Campos, Miyake e Bradshaw (1992) observaram que bebês japoneses e americanos exibiram, basicamente, as mesmas expressões emocionais. Houve uma diferença cultural na latência das expressões emocionais negativas, com os americanos respondendo mais rapidamente que os japoneses a um procedimento que envolvia a contenção do seu braço. A reação emocional das crianças parecia indicar uma maior contenção de manifestações por parte dos bebês japoneses.

Ainda no que se refere à influência do contexto sobre o desenvolvimento emocional da criança, Garrett-Peters e Fox (2007) realizaram um estudo com 117 crianças residentes em uma cidade metropolitana de Washington, sendo 59 de descendência chinesa e 58 europeias, com idades entre quatro e sete anos. O estudo propôs-se a examinar diferenças interculturais nos comportamentos de expressão emocional dos sujeitos em uma situação de desapontamento. Considerando que a cultura chinesa defende o controle emocional e, a denominada europeia, a individualidade e a autoexpressão, os pesquisadores esperavam que as crianças nascidas em países ocidentais demonstrassem mais comportamentos de expressão emocional do que as oriundas deste típico país oriental. Embora esta hipótese tenha sido apoiada pela expressividade das crianças, padrões interessantes surgiram no que diz respeito às expressões positivas e negativas. As crianças de descendência europeia e mais velhas demonstraram comportamentos mais positivos do que as crianças chinesas e mais novas.

Ao estudar o desenvolvimento emocional na perspectiva do Nicho Desenvolvimental (Harkness \& Super, 1992; 1994), é importante observar o lugar e a relevância das metas de socialização na dinâmica de inter-relação entre os seus subsistemas, e, na sequência, sua possível associação com as variáveis estudadas. 
Desse modo, as metas de socialização da emoção se referem a valores e ideias que os pais possuem sobre as emoções, que se concretizam em práticas e estratégias de socialização ao longo do desenvolvimento do filho. Isso significa que os objetivos que o pai e/ou a mãe estabelecem em termos do desenvolvimento emocional e da promoção da autoestima dos seus filhos, baseiamse no contexto e na cultura em que vivem (Ho, 2014; Keller \& Otto, 2009; Mendes \& Cavalcante, 2014). Associadas a estas crenças e metas de socialização voltadas para emoções, podem ser pensadas as relativas a duas necessidades humanas essenciais, quais sejam, as de autonomia e relação ou proximidade interpessoal, que impactam o desenvolvimento do self da criança. Visto de modo agregado, essas metas e crenças, ou etnoteorias parentais (Harkness \& Super, 1996), são forças importantes que moldam o desenvolvimento global da criança. Importa destacar, contudo, que tais objetivos parentais reproduzem modelos de orientações gerais historicamente condicionados por padrões culturais os mais diversos, relacionados à reprodução e ao cuidado infantil, que estabelecem metas destinadas a privilegiar e aperfeiçoar certas potencialidades humanas no desenvolvimento, em detrimento de outras (LeVine et al., 1996).

Com base nos estudos de Keller et al. (2006) e Keller (2007) envolvendo mães de diferentes contextos socioeconômicos e culturais, pode-se afirmar que as metas parentais evocam três modelos de orientações gerais para o desenvolvimento do self: interdependente, autônomo ou independente e autônomo-relacionado. $\mathrm{O}$ primeiro modelo corresponde à construção de um self conectado a outras pessoas, buscando manter a harmonia e o equilíbrio nas trocas sociais estabelecidas. Este modelo parece ser típico de ambientes rurais com a economia de subsistência. O segundo está relacionado a um self único e distinto, em que se priorizam a automaximização, realização pessoal e independência. Ele parece próprio de culturas urbanas (Seidl-deMoura et al., 2013). Por fim, o modelo autonomorelacional que representa um misto dos dois anteriores. Este último modelo foi descrito por Kağitçibaşi (2007) como uma orientação que engloba autonomia e relação, em que o self é definido como autônomo quanto à sua ação e relacional quanto à proximidade interpessoal (Keller et al., 2006; Keller, 2007; Lordelo et al., 2012; Mendes \& Cavalcante, 2014).

Tomando como referência os três modelos de orientação, Mendes e Cavalcante (2014) realizaram um estudo com 120 participantes (mães, babás e cuidadoras de creche), o qual buscou investigar a presença de metas de socialização relacionadas ao desenvolvimento emocional infantil, associando-as à valorização dos pais por trajetórias de desenvolvimento de selves mais autônomos ou mais interdependentes para seus filhos. Com base nos resultados obtidos, constatou-se que o conjunto de participantes apresentou uma tendência à valorização tanto da autonomia quanto da interdependência nas crianças. Nesse sentido, as autoras concluem que as participantes da pesquisa tinham majoritariamente a expectativa de que suas crianças viessem a satisfazer tanto necessidades e desejos individuais, quanto valorizassem aspectos relacionais, como por exemplo, manterem-se ligadas de modo mais próximo às pessoas com quem convivem, nos moldes em que discute Keller (2007).

O estudo de Mendes e Cavalcante (2014) constatou que $60 \%$ do grupo de mães consideram que crianças devem aprender a controlar as emoções durante os três primeiros anos, ao passo que, no grupo de outras cuidadoras, $60 \%$ destas não pensavam da mesma forma. A valorização de um maior controle emocional parece característica de grupos em que a interdependência é mais valorizada. Além disso, todas as participantes fizeram referência à importância das crianças sorrirem, sendo que mais de $90 \%$ afirmaram que é mais importante que sorriam para manifestarem sua emoção do que para se mostrarem simpáticas com as pessoas. Esta maneira de pensar pode ser vista como indicativa de uma tendência para a valorização de um self autônomo, com viés individualista.

Em um estudo realizado por Chan (2011), objetivou-se identificar a relação entre a orientação de self defendida pelas mães e as metas de socialização emocional que possuem para o futuro de seus filhos. Os dados foram coletados a partir de 189 mães chinesas que residem em Hong Kong. Os resultados sugeriram que a maioria das participantes possui orientação autônomo-relacional de self (Kağitçibaşi, 2007) em consequência das mudanças ocorridas no sistema econômico de Hong Kong (passou de essencialmente primário para o nível terciário). Além disso, o governo impôs educação de nove anos gratuita e obrigatória. Desse modo, a autonomia tornou-se gradualmente uma espécie de "bem pessoal", sendo cada vez mais valorizada. Em contrapartida, apesar do valor cultural do relacionamento interpessoal ainda permanecer evidente, a dependência intergeracional se tornou psicológica ao invés de material.

Em relação às metas maternas, os autores (Chan, 2011; Kağitçibaşi, 2007) ressaltaram que a competência emocional relacional esteve relacionada com o incentivo da construção de relações harmoniosas com as pessoas. Quando a competência emocional autônoma foi citada pelas mães, ressaltaram-se valores 
ocidentais, tais como a independência, a assertividade e a expressão de sentimentos e opiniões.

Para Ambrose (2013) as mães afetam o desenvolvimento emocional da criança, através da própria expressividade da emoção. Segundo a autora, isso ocorre de várias maneiras: a) por meio de processos diretos, como imitação e contágio; b) pode ser um correlato de outros comportamentos maternos que afetam a socialização da emoção; c) através da forma como efetivamente as crianças são capazes de interpretar e compreender as reações emocionais dos outros; d) por moldar seus pensamentos e sentimentos sobre si mesmo, dos outros e do mundo social (Ambrose, 2013; Benincá \& Gomes, 1998).

Considerando que as mães possuem papel fundamental na socialização e no desenvolvimento emocional da criança, este estudo procurou conhecer os valores e as crenças que orientam as metas e práticas maternas envolvidas na socialização da emoção. Por meio da sua realização, procurou-se investigar as expectativas e os objetivos relacionados ao desenvolvimento emocional da criança por mães residentes em município do interior do estado do Pará, e, na sequência, relacionar as metas de socialização emocional observadas aos aspectos que configuram os modelos de orientação sociocultural estudados no contexto pesquisado.

\section{Método}

\section{Delineamento da pesquisa}

O presente estudo é descritivo e possui caráter exploratório. Foi desenvolvido como parte dos objetivos propostos por um projeto maior que conta com apoio da FAPERJ e do CNPq, intitulado "Crenças e metas parentais de socialização das emoções em crianças".

\section{Participantes}

Participaram deste estudo 40 mães residentes em um município do interior do estado do Pará, que tinham, pelo menos, um filho, independentemente do sexo.

\section{Contexto da pesquisa}

O município, o qual foi realizada a pesquisa, corresponde a um pequeno lugarejo de aproximadamente 70 quilômetros a leste de Belém. Pode ser descrito como um contexto caracterizado como socioeconomicamente rural, que apresenta uma baixa densidade populacional, estimado em 12.000 habitantes, com várias famílias numerosas que convivem de maneira próxima. Em geral, o nível de instrução dos moradores é baixo (não alfabetizado a Ensino Fundamental Completo) e as famílias tiram do trabalho agrícola o seu sustento, assim como pela realização de atividades ligadas ao comércio ou em fábricas que se localizam no entorno do núcleo urbano.

\section{Ambiente da coleta}

O contato com as mães e a coleta dos dados foram realizados na sala de espera de uma Unidade Básica de Saúde, localizada em uma das mais importantes ruas do município. A sala de espera deste posto de saúde possui um espaço físico amplo e arejado, com banheiros, farmácia, cadeiras enfileiradas ao centro e uma recepção para informações e entrega de documentos.

\section{Instrumentos}

Os instrumentos utilizados para a coleta dos dados foram os seguintes:

- Formulário de Identificação dos Participantes FIP (Mendes \& Pessôa, 2013): contém 11 itens que levantam dados pessoais da criança (nome e data de nascimento) e dos pais (nome, data de nascimento, local onde reside e contato);

- Formulário de Dados Sociodemográficos - FDSD (Mendes, Pontes, Silva, Bucher-Maluschke, Reis, \& Baia-Silva, 2008; Freire, Silva, Moura, Pontes \& Araújo, 2014): possui 20 itens que investigam dados referentes aos pais (idade, escolaridade, estado civil, profissão, local em que nasceu e/ou foi criado, a idade que tiveram a criança, se tem outros filhos e em quantas horas por dia permanece com o filho) e à criança (idade e sexo);

- Questionário de Metas de Socialização da Emoção - QMSE: Composto de quatro perguntas estruturadas e abertas sobre o tema, que indagam, por exemplo, quais as características emocionais desejadas para o futuro do filho, quais as condições necessárias para o desenvolvimento das características mencionadas, que participação pensa que pode ter para que estas características sejam desenvolvidas/alcançadas, e ainda como pensam que elas surgem e em que época da vida. Este instrumento foi elaborado pela Prof - Dr ${ }^{\mathrm{a}}$ - Deise Mendes para um projeto maior que conta com apoio da FAPERJ e do CNPq, intitulado "Crenças e metas parentais de socialização das emoções em crianças".

\section{Procedimentos e considerações éticas}

A coleta dos dados foi aprovada pelo Comitê de Ética da Universidade do Estado do Rio de Janeiro, sob o Protocolo no 010.3.2010, que prevê a realização da pesquisa em dois contextos (urbano e não-urbano). 
Posteriormente, fez-se contato com a administração da Unidade Básica de Saúde, com o intuito de explanar os objetivos do estudo e obter a autorização do órgão para a realização da pesquisa. Quando autorizada, foram iniciadas as visitas à instituição para colher os dados.

As mães, presentes na sala de espera, foram abordadas, individualmente, e receberam informações orais e escritas sobre os objetivos da pesquisa, método empregado e direito de se recusar a participar do projeto.

A inclusão na amostra foi condicionada a mães residentes no município do interior do estado do Pará, que tinham, pelo menos, um filho, independentemente do sexo, além disso, que assinassem o Termo de Consentimento Livre e Esclarecida (TCLE).

\section{Análise dos dados}

As informações obtidas a partir da aplicação dos instrumentos foram organizadas em um banco de dados, por meio do Software SPSS 20. Além disso, as respostas das mães às questões abertas foram lidas na íntegra, buscando identificar as categorias de análise, de acordo com definições previamente construídas. Tais categorias foram inspiradas em outras, adaptadas para estudo com população brasileira por Seidl-de-Moura et al. (2008) que, por sua vez, com base em Harwood (1995) e Leyendecker et al. (2002). Com isso, esperavase aprofundar a compreensão das possíveis variações entre as metas de socialização emocional das mães, bem como explorar a relação destas com aspectos que caracterizam os diferentes modelos de orientação sociocultural (ora características que valorizam a autonomia, ora a relação, ou mesmo, ambas), conforme proposto por Keller (2007) e Keller et al. (2006).

Foram realizados cálculos descritivos (frequência, média, desvio padrão), através do Software SPSS 20, para informar sobre as variáveis sociodemográficas, caracterizando o conjunto de mães participantes, neste sentido. Para descrever as metas de socialização emocional mencionadas pelas mães e suas condicionantes, realizou-se primeiramente uma análise do conteúdo das respostas maternas, considerando as categorias definidas anteriormente (ver Tabelas 1, 2 e 3), que foram organizadas de acordo as questões contidas no Questionário de Metas de Socialização da Emoção. Especificamente para identificar a presença e pesos relativos das diversas categorias trabalhadas, foi feito o cálculo da porcentagem com base na frequência com que estas foram mencionadas. No passo seguinte, calculou-se a porcentagem e o número de vezes em que as metas de socialização da emoção foram mencionadas de acordo com a idade e a escolaridade da mãe.

\section{Resultados}

O presente item está organizado em duas partes. Na parte I, apresentam-se a frequência, a média e o desvio padrão dos resultados obtidos para caracterizar, sociodemograficamente, as mães participantes do estudo. Na parte II, estão dispostos os resultados referentes às metas maternas de socialização emocional a fim de identificar as características emocionais que as mães desejam para o futuro dos filhos, as condições necessárias para o desenvolvimento das características mencionadas e o papel da atuação parental no desenvolvimento destas.

\section{Características sociodemográficas}

Um total de 40 mães que participaram do presente estudo, a faixa etária variou entre 13 e 36 anos $(M=23,8$ anos, $D P=5,2)$ e, durante a coleta, a maioria $(47,5 \%)$ referiu ter um único filho. A idade das crianças variou entre 1 e 35 meses ( $M=15,73$ meses; $D P=8,4)$, sendo a maior parte do sexo masculino $(57,5 \%)$. Quanto ao nível de escolaridade, majoritariamente as mães tinham o ensino médio incompleto (50\%) ou completo (27,5\%). Com relação ao estado civil, $82,5 \%$ referiram estar casadas ou em união estável.

Outro aspecto importante a ser ressaltado é que houve o predomínio de mães que não exerciam atividade remunerada $(67,5 \%)$, realizavam unicamente atividades reconhecidas como afazeres domésticos. Em razão disso, a maioria delas $(97,5 \%)$ declarou destinar a maior parte do tempo disponível na criação dos filhos. Com base nos dados contidos nos questionários aplicados, constatou-se ainda que a maioria das mães nasceu no município de Castanhal (37,5\%) e foi criada no mesmo local $(42,5 \%)$.

\section{Metas maternas de socialização emocional}

Os resultados sobre as metas de socialização emocional maternas serão apresentados de acordo com a ordem das questões (1, 2 e 3 ) contidas no Questionário de Metas de Socialização da Emoção. Cada questão está dividida em três seções para análise dos dados. Na primeira seção, apresentam-se resultados do cálculo da porcentagem com base na frequência de vezes que as respostas das mães estiveram relacionadas às categorias predefinidas (ver Tabelas 1,2 e 3). Na segunda, apresentam-se os resultados referentes à porcentagem e o número de vezes que as mães mencionaram as categorias de cada questão, de acordo com a idade e escolaridade destas. Por fim, na terceira seção, foram transcritos trechos das entrevistas com as mães que evidenciam as metas de socialização por elas mencionadas. 
TABELA 1

Síntese do quadro de categorias em relação às características emocionais desejadas pelas mães (Questão 1)

\section{CATEGORIA}

Automaximização

Preocupação com que a criança se torne emocionalmente autoconfiante e independente, e que desenvolva totalmente seus talentos e capacidades ligadas à esfera emocional.

Autocontrole

Preocupação com que a criança desenvolva a capacidade de regulação emocional, especialmente as indesejáveis, de impulsos negativos, controlando a manifestação exacerbada de emoções.

Emotividade

Preocupação com que a criança desenvolva a capacidade para intimidade emocional com outros, e que seja amada; seja capaz de iniciar e manter relacionamentos afetivos, com trocas e manifestações de afeto.

Expectativas sociais

Preocupação com que a criança atenda a expectativas sociais quanto às regras de exibição de suas emoções e sentimentos e de reação às manifestações emocionais e comportamentos das outras pessoas.

Não classificável nas categorias predefinidas

Não respondeu

\section{EXEMPLOS}

Que saiba expressar suas emoções de forma satisfatória; que seja alegre; segura; feliz; emocionalmente segura de si e autoconfiante.

Que use a razão nos sentimentos; que seja equilibrada; que não se exalte toda vez que não conseguir o que quer; que não se abale com qualquer problema.

Que tenha compaixão das outras pessoas; que seja generosa; amigável; sensível; gentil; amável; meiga; que se preocupe com as outras pessoas.

Que saiba se relacionar bem em sociedade.

A resposta não se aplica a nenhuma das definições de categoria estabelecidas. Não sabe ou não quis responder.

TABELA 2

Síntese do quadro de categorias em relação às condições para o desenvolvimento de características emocionais desejadas pelas mães (Questão 2)

\begin{tabular}{ll}
\hline CATEGORIA & EXEMPLO \\
\hline $\begin{array}{l}\text { Centradas na mãe (ou cuidador) } \\
\text { Preocupação em ensinar a criança a partir da disciplina, do aconselhamento } \\
\text { e/ou através de exemplos diários. }\end{array}$ & $\begin{array}{l}\text { Ser modelo ou oferecer modelos; disciplinar; aconselhar; ensinar por } \\
\text { demonstração ou participação. }\end{array}$ \\
$\begin{array}{l}\text { Centradas no contexto } \\
\begin{array}{l}\text { Preocupação em oferecer para a criança um ambiente benéfico para o seu } \\
\text { desenvolvimento emocional, proporcionando relações interpessoais positivas }\end{array}\end{array}$ & Oferecer boas oportunidades sociais; dar educação de qualidade. \\
e uma educação de qualidade. & $\begin{array}{l}\text { Centradas na criança } \\
\text { Características ou capacidades inerentes à criança. }\end{array}$ \\
$\begin{array}{l}\text { Não se aplica } \\
\text { Ao mencionar, que a criança se desenvolve naturalmente, sem que seja ne- } \\
\text { cessário vivenciar situações para que ocorra o desenvolvimento emocional. }\end{array}$ & $\begin{array}{l}\text { Não há desenvolvimento, já que as características são consideradas inatas ou } \\
\text { fruto de maturação biológica. } \\
\text { Não classificável nas categorias predefinidas }\end{array}$ \\
Não respondeu & A resposta não se aplica a nenhuma das definições de categoria estabelecidas. \\
\hline
\end{tabular}

TABELA 3

Síntese do quadro de categorias em relação à atuação parental para o desenvolvimento de características emocionais desejadas pelas mães (Questão 3)

\begin{tabular}{|c|c|}
\hline CATEGORIA & EXEMPLO \\
\hline $\begin{array}{l}\text { Educar/Orientar } \\
\text { Preocupação em ensinar a criança a partir da disciplina, do aconselhamento } \\
\text { e/ou através de exemplos diários. }\end{array}$ & $\begin{array}{l}\text { Ser modelo ou oferecer modelos; ensinar por participação, explicações } \\
\text { verbais, ou medidas disciplinares. }\end{array}$ \\
\hline $\begin{array}{l}\text { Manter relações de proximidade pessoal } \\
\text { Preocupação em proporcionar, para a criança, apoio afetivo, pessoal e } \\
\text { familiar, no sentido de oferecer carinho e cuidado ao filho. }\end{array}$ & Fazer-se afetivamente presente para a criança; acompanhar; dar amor. \\
\hline $\begin{array}{l}\text { Prover condições materiais e sociais } \\
\text { Preocupação em oferecer para a criança condições favoráveis de educação, } \\
\text { sustento e saúde. }\end{array}$ & $\begin{array}{l}\text { Oferecer boas oportunidades sociais; dar educação de qualidade; prover o } \\
\text { sustento da criança. }\end{array}$ \\
\hline $\begin{array}{l}\text { Não se aplica } \\
\text { Ao mencionar, que a criança se desenvolve naturalmente, sem que seja ne- } \\
\text { cessário vivenciar situações para que ocorra o desenvolvimento emocional. }\end{array}$ & $\begin{array}{l}\text { Não há o que fazer, não há desenvolvimento, já que as características são } \\
\text { consideradas inatas ou fruto de maturação biológica. }\end{array}$ \\
\hline Não classificável nas categorias predefinidas. & A resposta não se aplica a nenhuma das definições de categoria estabelecidas. \\
\hline Não respondeu & Não sabe ou não quis responder. \\
\hline
\end{tabular}


A Tabela 4 traz dados que se reportam às respostas conferidas pelas mães quando perguntadas na questão 1: "Que características emocionais você desejaria que seu filho(a) tivesse como adulto?". Como observado, as metas relacionadas à automaximização $(47,7 \%)$ foram as mais mencionadas pelas mães, seguidas das metas de emotividade (19,3\%) e autocontrole $(6,4 \%)$. Do mesmo modo, verificou-se que estas metas foram mais citadas pelas mães com a faixa etária entre 19 e 29 anos e as que tinham nível médio. Por sua vez, constatou-se que mães que possuíam o ensino superior mencionaram apenas metas relacionadas à automaximização.

Ao referir metas relacionadas à automaximização, as mães foram enfáticas ao mencionar o desejo de que, no futuro, o filho seja uma pessoa feliz. A felicidade foi atrelada, com frequência, à autoconfiança na manifestação e na vivência de suas emoções e sentimentos, além de destacarem a capacidade dele ou dela vir a desenvolver de modo saudável suas capacidades emocionais como indivíduo autônomo. Quanto às metas referentes à categoria de emotividade, as mães enfatizaram, principalmente, a importância da amabilidade e da afetividade ao próximo, sendo estas características ligadas à preocupação de que sua criança desenvolva a capacidade de expressar intimidade emocional com outros e seja capaz de iniciar e manter relacionamentos afetivos, com trocas e manifestações de afeto.

Pela Tabela 5, observa-se que quando perguntado às mães na questão 2: "O que você acha que é necessário para que ele(a) desenvolva/tenha essas características?", houve um maior número de respostas relacionadas à categoria centradas na mãe $(44,7 \%)$, e, por sua vez, as metas centradas na criança $(19,6 \%)$ foram menos referidas pelas participantes. Ainda de acordo com a Tabela 5, verificou-se que as mães com faixa etária entre 19 e 29 anos, e as que apresentavam como escolaridade o nível médio, citaram com maior frequência que as práticas de cuidado devem estar centradas na mãe, possivelmente com a finalidade de que suas metas de socialização emocional sejam atingidas.

A Tabela 6 apresenta exemplos de relatos das mães em relação à preocupação de exercer um papel ativo na criação dos filhos, ensinando à criança a partir da disciplina, do aconselhamento e de exemplos diários.

$\mathrm{Na}$ Tabela 7 está demonstrada a porcentagem e a frequência das respostas dadas à questão 3: "O que você pensa que pode fazer para que ele(a) possa desenvolver/ter essas características?". Nesta questão que se refere à atuação parental para o desenvolvi-

TABELA 4

Porcentagem e o número de vezes em que as metas de socialização da emoção foram mencionadas de acordo com a idade e escolaridade da mãe (Questão 1)

\begin{tabular}{|c|c|c|c|c|c|c|}
\hline Idade e Escolaridade & Automaximização & Autocontrole & Emotividade & $\begin{array}{c}\text { Expectativas } \\
\text { sociais }\end{array}$ & $\begin{array}{c}\text { Não classificável } \\
\text { nas categorias } \\
\text { predefinidas }\end{array}$ & Não respondeu \\
\hline \multicolumn{7}{|l|}{ Idade } \\
\hline Até 18 anos & $10 \%(11)$ & & $0,9 \%(1)$ & $0,9 \%(1)$ & $7,3 \%(8)$ & \\
\hline 19-29 anos & $32,1 \%(35)$ & $3,7 \%(4)$ & $9,2 \%(10)$ & $0,9 \%(1)$ & $13,8 \%(15)$ & \\
\hline $30-36$ anos & $5,5 \%(6)$ & $2,8 \%$ & $9,2 \%(10)$ & & $3,7 \%(4)$ & \\
\hline \multicolumn{7}{|l|}{ Escolaridade } \\
\hline Ensino Fundamental & $1,8 \%(2)$ & & $1,8 \%(2)$ & & $1,8 \%(2)$ & \\
\hline Ensino Médio & $36,7 \%(40)$ & $6,4 \%(7)$ & $17,5 \%(19)$ & $1,8 \%(2)$ & $21,2 \%(23)$ & \\
\hline Ensino Superior & $9,2 \%(10)$ & & & & $1,8 \%(2)$ & \\
\hline
\end{tabular}

TABELA 5

Número de vezes em que foram mencionadas as condições para o desenvolvimento emocional pesquisadas, de acordo com a idade e escolaridade da mãe (Questão 2)

\begin{tabular}{|c|c|c|c|c|c|c|}
\hline Idade e Escolaridade & Centradas na mãe & $\begin{array}{c}\text { Centradas no } \\
\text { contexto }\end{array}$ & $\begin{array}{c}\text { Centradas na } \\
\text { criança }\end{array}$ & Não se aplica & $\begin{array}{c}\text { Não classificável } \\
\text { nas categorias } \\
\text { predefinidas }\end{array}$ & Não respondeu \\
\hline \multicolumn{7}{|l|}{ Idade } \\
\hline Até 18 anos & $8,9 \%(5)$ & $5,4 \%(3)$ & & & & \\
\hline $19-29$ anos & $25 \%(14)$ & $10,7 \%(6)$ & $17,9 \%(10)$ & & $7,1 \%(4)$ & $1,8 \%(1)$ \\
\hline $30-36$ anos & $10,7 \%(6)$ & $7,1 \%(4)$ & $1,8 \%(1)$ & & $1,8 \%(1)$ & $1,8 \%(1)$ \\
\hline \multicolumn{7}{|l|}{ Escolaridade } \\
\hline Ensino Fundamental & $3,6 \%(2)$ & $3,6 \%(2)$ & & & & \\
\hline Ensino Médio & $41,1 \%(23)$ & $17,8 \%(10)$ & $10,7 \%(6)$ & & $8,9 \%(5)$ & $1,8 \%(1)$ \\
\hline Ensino Superior & & $1,8 \%(1)$ & $8,9 \%(5)$ & & & $1,8 \%(1)$ \\
\hline
\end{tabular}


mento de características emocionais desejadas, a maior parte das mães referiu que é necessário educar / orientar $(63,7 \%)$ a criança, seguidas pela preocupação em manter relações de proximidade pessoal $(27,5 \%)$ e prover condições materiais e sociais. Constatou-se, também, que as mães com faixa etária entre 19 e 29 anos e mães com o nível médio, consideram que se deve educar/orientar as crianças quanto às emoções.
No que se refere às categorias de atuação parental para o desenvolvimento de características emocionais desejadas (Tabela 8), a maioria das participantes destacou a importância de educar e orientar a criança, sendo ou oferecendo modelos, disciplinando, aconselhando, conversando, ensinando por demonstração, participando, oferecendo explicações verbais e medidas disciplinares.

TABELA 6

Categorias em relação ao que deve ser feito para que as metas maternas sejam cumpridas tomando como referência as categorias predefinidas (Questão 2)

\begin{tabular}{ll}
\hline CATEGORIA & EXEMPLOS (Mães) \\
\hline Centradas na mãe & M06 - “(..) Conversar sobre o que é certo e o que é errado" \\
& M16 - "Estar sempre presente ensinando a fazer as coisas" \\
Centradas no contexto & M01 - "Viver em um ambiente tranquilo e sossegado" \\
& M17 - "Tem uma boa convivência" \\
Centradas na criança & M21 - "Conviver com a família" - "Aprender a demonstrar e saber compreender os sentimentos" \\
& M06 - "Saber brincar (...)" \\
Não classificável nas categorias predefinidas & M19 - "Ser obediente e saiba lhe dar com as pessoas" \\
& M11 - "Manter a família" \\
Não respondeu & M14 - "Ter uma direção espiritual” \\
Não se aplica & M24, M26 \\
\hline
\end{tabular}

TABELA 7

Número de vezes em que as mães mencionaram o papel da atuação parental no desenvolvimento emocional de acordo com a idade e escolaridade da mãe (Questão 3)

\begin{tabular}{|c|c|c|c|c|c|c|}
\hline Idade/Escolaridade & Educar/Orientar & $\begin{array}{c}\text { Manter relações } \\
\text { de proximidade } \\
\text { pessoal }\end{array}$ & $\begin{array}{l}\text { Prover condições } \\
\text { materiais e sociais }\end{array}$ & Não se aplica & $\begin{array}{c}\text { Não classificável } \\
\text { nas categorias } \\
\text { predefinidas }\end{array}$ & Não respondeu \\
\hline \multicolumn{7}{|l|}{ Idade } \\
\hline Até 18 anos & $12,5 \%(10)$ & $2,5 \%(2)$ & & & & \\
\hline 19-29 anos & $36,3 \%(29)$ & $21,3 \%(17)$ & $5 \%(4)$ & & $2,5 \%(2)$ & \\
\hline 30-36 anos & $15 \%(12)$ & $3,7 \%(3)$ & & & $1,2 \%(1)$ & \\
\hline \multicolumn{7}{|l|}{ Escolaridade } \\
\hline Ensino Fundamental & $5 \%(4)$ & $1,2 \%(1)$ & & & & \\
\hline Ensino médio & $53,8 \%(43)$ & $25 \%(20)$ & $5 \%(4)$ & & $2,5 \%(2)$ & \\
\hline Ensino superior & $5 \%(4)$ & $1,2 \%(1)$ & & & $1,2 \%(1)$ & \\
\hline
\end{tabular}

TABELA 8

Categorias para atuação parental em prol do desenvolvimento de características emocionais desejadas

\begin{tabular}{ll}
\hline CATEGORIA & EXEMPLOS (MÃES) \\
\hline Educar/Orientar & M003- "Conversar mais sobre os sentimentos" \\
& M005 - "Ensinando e fazendo o que ela tem que aprender" \\
& M018 - "Tenho que educar bem e dar um bom exemplo para ela" \\
Manter relações de proximidade pessoal & M021 - "Conviver com o meu filho" \\
& M029 - "(..) ser companheira, presente e carinhosa com a criança" \\
Prover condições materiais e sociais & M030 - "Mostrar carinho, compreensão e respeito" \\
& M001- "Oferecer uma boa educação" \\
Não classificável nas categorias predefinidas & M012 - "Por em um bom estudo (...) dar boas condições (...)" \\
& M014 - "Orientá-lo na direção espiritual" \\
Não respondeu & M026 - "Realizar os sonhos dele" \\
Não se aplica & -
\end{tabular}




\section{Discussão}

Com base no perfil sociodemográfico das mães residentes no contexto estudado, assume-se que estas apresentam tendência a adotar de forma predominante a orientação relacional. Foram identificadas características emocionais que estas desejam que seus filhos apresentem no futuro, as condições necessárias para o desenvolvimento das características mencionadas e o papel da atuação parental no desenvolvimento delas. Para isso, organizou-se a discussão a partir da ordem em que as questões foram apresentadas no questionário (1, 2 e 3). Cada questão foi dividida em três seções para a discussão dos dados.

Assim, no que diz respeito à questão 1 deste estudo, constatou-se que a maioria das mães mencionou metas relacionadas à automaximização, a qual se refere de modo mais direito a características de um indivíduo autoconfiante na manifestação e na vivência das emoções e sentimentos, características relacionadas ao modelo de orientação autônomo. Embora em menor porcentagem, as mães também referiram metas relacionadas à categoria emotividade, a qual está diretamente relacionada com o modelo de orientação relacional. Dessa maneira, com base na literatura (Keller et al., 2006; Keller, 2007), é possível sugerir que esta comunidade está iniciando um período de transição do modelo de orientação relacional para o autônomorelacionado, previsto por Kağitçibaşi (2007). Supõe-se que esta comunidade esteja incorporando elementos constituintes do modelo autônomo de maneira a somar às características relacionais já existentes. De acordo com Lordelo et al. (2012), esta transição de um modelo relacional para o autônomo-relacionado é uma tendência de países em desenvolvimento, como por exemplo, o Brasil, que possui uma crescente escolarização e industrialização mais recente. Lamm et al. (2008) complementam esta ideia, afirmando que, na era da globalização crescente, essas mudanças em direção a uma maior valorização da independência em detrimento da interdependência, podem ser esperadas em ambientes culturais os mais diversos, sendo esta uma tendência em boa parte do mundo urbanizado, presente na contemporaneidade.

Pode ser pensada uma correspondência entre tal visão e os resultados obtidos quanto às metas relacionadas à automaximização e à emotividade, as categorias mais citadas pelas mães na faixa etária de 19 e 29 anos, e pelas que tinham nível médio. De modo diverso, foi observado que as mães com ensino superior mencionaram apenas metas relacionadas à automaximização. Benincá e Gomes (1998), anos antes, já discutiram sobre o processo histórico e social pelo qual a agricultura e a produção familiar cedem gradativamente lugar ao interesse por profissões liberais para os homens, e ao magistério para as mulheres. Em consequência deste processo, a mãe, ao acrescentar, cada vez mais cedo, ao trabalho doméstico e ao casamento, uma nova prioridade, ou seja, a formação escolar, impulsiona o crescimento da consciência pela profissionalização feminina, o contato direto com os avanços tecnológicos, além de abrir novas possibilidades sobre o mercado de trabalho e a competitividade.

De acordo com os estudos empíricos relatados na literatura (Benincá \& Gomes, 1998; Keller et al., 2008; Seidl-de-Moura et al., 2008; Vieira et al., 2010), pode-se dizer que os aspectos socioeconômicos repercutem na vida das famílias e dos indivíduos e, mais especificamente, nas metas de socialização dos pais em relação aos seus filhos, conforme previsto pelo modelo teórico de Kağitçibaşi (2007). Assim, tomando como referência esses estudos, compreende-se que na população estudada a escolaridade da mãe pode ser tomada como uma importante fonte de variação na formulação das metas de socialização, tendo em vista que os resultados apontam que mães de maior escolaridade mencionaram mais metas de autonomia (automaximização) do que relacionais (emotividade).

Qualitativamente, ao mencionarem metas de socialização emocional relacionadas diretamente à automaximização, as mães demonstraram preocupação em que a criança se torne emocionalmente autoconfiante e independente, e que desenvolva totalmente seus talentos e capacidades ligadas à esfera emocional. Isto implica tornar-se autoconfiante na manifestação e na vivência de suas emoções e sentimentos, e desenvolver de modo saudável suas capacidades emocionais como indivíduo autônomo. Ao mesmo tempo, em que, apontaram frequentemente metas relacionadas à emotividade, as mães demonstraram preocupação com que a criança desenvolvesse a capacidade para intimidade emocional com outros, e que fosse amável, além de ser capaz de iniciar e manter relacionamentos afetivos, com trocas e manifestações de afeto.

No que se refere à questão 2 , constatou-se um maior número de respostas relacionadas à categoria centradas na mãe $(44,7 \%)$, sendo que as mães que tinham nível médio de escolaridade foram as que referiram de modo preferencial estratégias centradas nelas (Tabela 8). Este resultado está em consonância com o que documenta a literatura (Benincá \& Gomes, 1998), que considera que a mãe desempenha papel fundamental nas rotinas de cuidado e na educação infantil. Além de garantir a sobrevivência do bebê contribui para a socialização do(a) filho/filha, sendo esta uma premissa 
que tem presença em diversas sociedades e culturas. Em vista disso, considera-se que a mãe é a primeira agente socializadora da criança, a qual, por meio de sinais sutis e implícitos, comunica para o(a) filho(a) formas de ser e agir valorizadas em seu ambiente cultural. Passa a contribuir, desse modo, para a adaptação da criança em relação ao ambiente em que vive (Martins, 2014).

Com base nos resultados obtidos, constatou-se ainda que as mães na faixa etária de 19 e 29 anos, e as que tinham nível médio escolar, citaram em maior número que as condições para o desenvolvimento de características emocionais desejadas estão centradas nelas mesmas, ressaltando a preocupação em ensinar a criança a partir da disciplina, do aconselhamento ou até mesmo através de exemplos diários. Tal evidência torna ainda mais clara a importância fundamental da mãe no processo de socialização emocional da criança. Como ressaltam Seidl-de-Moura et al. (2008), as interações com a genitora possuem relevância significativa no desenvolvimento do self, do afeto e da cognição da criança.

A questão 3 remete à atuação parental para o desenvolvimento de características emocionais desejadas. Verificou-se que a maior parte das mães considera que é necessário Educar/Orientar (63,7\%) a criança, sendo esta uma atribuição sua, como responsável por sua guarda e criação. Constatou-se, ainda, que a maioria das mães que pensam desta forma encontra-se na faixa etária de 19 e 29 anos e/ou concluiu o nível médio. Elas admitem ser papel das mães oferecer à criança explicações verbais ou não verbais sobre as emoções, aconselhando, ajudando, orientando, incentivando, através de um ato de carinho, como por exemplo, dar um abraço, um beijo. Uma estratégia deste tipo pode favorecer que a criança incremente seu amadurecimento emocional, pois segundo Mendes e Cavalcante (2014), estas trocas afetivas que são estabelecidas com a criança e a maneira como as mães lidam com as emoções, tornam-se mais uma fonte significativa de referência e aprendizagem.

\section{Considerações Finais}

Este estudo buscou investigar as metas de socialização de emoção que expressam expectativas e objetivos relacionados ao desenvolvimento emocional da criança por mães residentes no município do interior do estado do Pará, tomando como referência teórica à noção de Nicho Desenvolvimental proposta por Harkness e Super (1992, 1994). Nessa direção, os resultados obtidos, estiveram em consonância com a literatura no que diz respeito ao papel fundamental que as mães desempenham no desenvolvimento emocional dos seus filhos.

Tomando como referência os modelos de orientação de self e algumas evidências deles derivadas (Kağitçibaşi, 2007; Keller et al., 2006; Keller, 2007; Mendes \& Cavalcante, 2014; Seidl-de-Moura et al. 2012) esperava-se que as mães residentes em um município do interior do estado do Pará enfatizassem como metas de socialização emocional as relacionadas às dimensões do modelo de interdependência. Entretanto, constatou-se que as mães privilegiaram as características de ambos modelos (autonomia e interdependência), pois, ao mesmo tempo em que defendem a autoconfiança na manifestação e na vivência das emoções, ressaltam a preocupação com que a criança desenvolva a capacidade para a intimidade emocional com outras pessoas. Este resultado sugere que o contexto escolhido está passando por um período transitório de mudança gradual do modelo de orientações gerais, apontando para a formação de uma nova síntese denominada de autônomo-relacionada (Kağitçibaşi, 2007).

Pode-se apontar como limitações deste estudo o número reduzido de mães participantes e o fato do ambiente de pesquisa, que foi escolhido, estar passando por um processo transitório, no que diz respeito às características sociodemográficas da população (nível socioeconômico, tamanho das famílias, entre outras). Por essa razão, é possível inferir que por se tratar de um contexto rural que está passando por mudanças na sua base produtiva (atividade econômica) e no perfil sociodemográfico da sua população, isso pode ser associado às mudanças no modelo de orientação (relacional) até então dominante.

Neste ponto, evidencia-se a necessidade de estudos adicionais utilizando o mesmo modelo teórico, mas com um número maior de participantes, para que seja possível explorar melhor as diferentes configurações associadas a variáveis sociodemográficas como idade da mãe e nível de escolaridade. Isso tornaria possível realizar análises mais específicas sobre as metas de socialização emocional em três gerações de mães provenientes de contextos com populações tradicionais, rurais ou não, como por exemplo, comunidades quilombolas e ribeirinhas. 


\section{Referências}

Ambrose, H. N. (2013). Young children's emotion regulation and social skills: the role of maternal emotional socialization and mother-child interactional synchrony. Electronic Theses and Dissertations. University of Windsor.

Benincá, C. R. S. \& Gomes, W. B. (1998). Relatos de mães sobre transformações familiares em três gerações. Estudos de Psicologia, 3(2), 177-205. https://doi.org/10.1590/S1413-294X1998000200002

Camras, L. A., Oster, H., Campos, J. J., Miyake, K, \& Bradshaw, D. (1992). Japanese and American infants' responses to arm restraint. Developmental PsycllOlogy, 28(4), 578-583. https://doi.org/10.1093/acprof:oso/9780195179644.003.0014

Chan, S. M. (2011). Mothers' construal of self and emotion socialisation goals. Early Child Development and Care, 181(5), 613-624. https://doi.org/10.1080/03004431003671820

Cole, P. M. \& Tamang, B. L. (1998). Nepali children's ideas about emotional displays in hypothetical challenges. Developmental Psychology, 34(4), 640. https://doi.org/10.1037//0012-1649.34.4.640

Ekman, P. \& Davidson, R. J. (1994). The nature of emotion: Fundamental questions. New York: Oxford University Press.

Freire, V. R. B. P., da Costa Silva, S. S., de Moura, M. L. S., Pontes, F. A. R., \& Araújo, M. E. C. (2014). Metas e expectativas parentais em contextos urbano e ribeirinho da Amazônia. Revista Interamericana de Psicologia / Interamerican Journal of Psychology, 48(1).

Garrett-Peters, P. T. \& Fox, N. A. (2007). Cross-cultural differences in children's emotional reactions to a disappointing situation. International Journal of Behavioral Development, 31(2), 161-169. https://doi.org/10.1177/0165025407074627

Halberstadt A. G. \& Lozada F. T. (2011) Culture and Emotions in the first 5 to 6 Years of Life. Topic emotions. In R. E. Tremblay, Boivin, M. \& Peters R. D. Encyclopedia on Early Childhood Development, 1-6.

Harkness, S. \& Super, C. M. (1992). Parental ethnotheories in action. In I. E. Siegel, McGillicuddy-DeLisi, A. V. \& Goodnow, J. J. Parental belief systems: The psychological consequences for children (pp. 373-391). https://doi. org/10.1016/0277-9536(94)90391-3

Harkness, S. \& Super, C. M. (1994). Developmental niche: A theoretical framework for analyzing the household production of health. Social Science and Medicine, 38(2), 219-226.

Harkness, S. \& Super C. M. (1996). Introduction. Em S. Harkness \& C. M. Super (Eds.). Parents' Cultural Belief Systems (pp. 1-23). New York: The Guilford Press.

Harwood, R. L. (1992). The influence of culturally derived values on Anglo and Puerto Rican mothers' perceptions of attachment behavior. Child Development, 63, 822-839. https://doi.org/10.2307/1131236

Ho, G. W. (2014). Acculturation and its implications on parenting for chinese immigrants a systematic review. Journal of Transcultural Nursing, 25(2), 145-158. https://doi.org/10.1177/1043659613515720

Instituto Brasileiro de Geografia e Estatística [IBGE] (2014). Censo demográfico 2010, Rio de Janeiro: IBGE. Recuperado em 23 de setembro de 2014. Obtido em http://www.censo2010.ibge.gov.br/

Kağitçibaşi, Ç. (2007). Family, self, and human development across cultures: theory and applications. Mahwah, NJ: Lawrence Erlbaum Associates.

Keller, H. (2007). Cultures of infancy. Mahwah, Nova Jersey: Erlbaum.

Keller, H., Demuth, C., \& Yovsi, R. D. (2008). The multi-voicedness of independence and interdependence: The case of the Cameroonian Nso. Culture \& Psychology, 14(1), 115-144. https://doi.org/10.1177/1354067X07082752

Keller, H. \& Otto, H. (2009). The cultural socialization of emotion regulating during infancy. Journal of Cross-Cultural Psychology, 40(6), 996-1011. https://doi.org/10.1177/0022022109348576

Keller, H. \& Kärtner, J. (2013). Development - The cultural solution of universal developmental tasks. In M. J. Gelfand, C. Chiu, \& Y. Hong (Eds.). Advances in Culture and Psychology (Vol. 3, pp. 63-116). New York, NY: Oxford University Press. https://doi.org/10.1093/acprof:oso/9780199930449.003.0002

Lamm, B., Keller, H., Yovsi, R. D., \& Chaudhary, N. (2008). Grandmaternal and maternal ethnotheories about early child care. Journal of Family Psychology, 22(1), 80. https://doi.org/10.1037/0893-3200.22.1.80

LeVine, A. R., Dixon, S., LeVine, S., Richman, A., Leiderman, P. H., Keefer, C., \& Brazelton, T. B. (1996). Child Care and Culture: Lessons from Africa. Cambridge University Press.

Leyendecker, B., Lamb, M. E., Harwood, R. L., \& Schölmerich, A. (2002). Mothers' socialisation goals and evaluations of desirable and undesirable everyday situations in two diverse cultural groups. International Journal of Behavioral Development, 26(3), 248-258. https://doi.org/10.1080/01650250143000030

Lordelo, E. R., Roethle, M., \& Mochizuki, A. B. (2012). Metas de socialização em diferentes contextos. Paidéia, 22(51), 33-42. https://doi.org/10.1590/S0103-863X2012000100005

Martins, G. D. F. M. (2014). Metas de socialização maternas e estilos de interação mãe-bebê no primeiro e no segundo ano de vida da criança. Tese de doutorado publicada. Programa de pós Graduação em Psicologia do Instituto de Psicologia da Universidade Federal do Rio Grande do Sul. Santa Catarina, RS.

Mendes, D. M. L. F. \& Pessôa, L. F. (2013). Comunicação afetiva nos cuidados parentais. Psicologia em Estudo, 18(1). https://doi.org/10.1590/S1413-73722013000100003

Mendes, D. M. L. F. \& Cavalcante, L. I. C. (2014). Modelos de self e expressão emocional em bebês: concepções de mães e outras cuidadoras. Psico, 45(1), 110-119. https://doi.org/10.15448/1980-8623.2014.1.13768 
Mendes, L. S. A., Pontes, F. A. R., Silva, S. S. C., Bucher-Maluschke, J. S. N. F., Reis, D. C., \& Baía-Silva, S. D. (2008). Inserção ecológica no contexto de uma comunidade ribeirinha amazônica. Revista Interamericana de Psicologia, 42(1), 1-10.

Seidl-de-Moura, M. L., Bandeira, T. T., de Marca, R. G. da C., Pessôa, L. F., Mendes, D. M. L. F., Vieira, M. L., et al. (2012). Self-recognition and self-regulation: the relationship with socialization trajectories and children's sex. Spanish Journal of Psychology, 15(2), 604-612. https://doi.org/10.5209/rev_SJOP.2012.v15.n2.38871

Seidl-de-Moura, M. L., Lordelo, E., Vieira, M. L., Piccinini, C. A., Siqueira, J. O., Magalhães, C. M. C., \& Rimoli, A. (2008). Brasilian mothers' socialization goals: Intracultural diferences in seven Brazilian cities. International Journal of Behavior Development, 32(6), 465-472. https://doi.org/10.1177/0165025408093666

Seidl-de-Moura, M. L., Mendes, D. M. L. F., Vieira, M. L., Korbarg, A. P., Pessôa, L. F., \& Bandeira, T. T. A. (2013). Brazilian mothers' description of their children: dimensions of autonomy and relatedness. Psicologia: Teoria $e$ Pesquisa, 29(3), 249-255. https://doi.org/10.5772/57083

Autores:

Bianca Reis Fonseca - Mestre, Universidade Federal do Pará

Lília Iêda Chaves Cavalcante - Pós-Doutora, Universidade Federal do Pará

Deise Maria Leal Fernandes Mendes - Doutora, Universidade do Estado do Rio de Janeiro.

\section{Endereço para correspondência:}

Bianca Reis Fonseca

Av. Dezesseis de Novembro, 881, apto. 302

66023-220 - Belém, PA, Brasil

<biancareisfonseca@gmail.com>

Recebido em: 04.10.2016

Aceito em: 14.06.2017 